\title{
Principles for Designing Systems of Screen Icons
}

\author{
Elena González-Miranda ${ }^{1}$ and Tania Quindós ${ }^{1}$ \\ ${ }^{1}$ Universidad del País Vasco/Euskal Herriko Unibertsitatea (UPV/EHU), \\ Departamento de Dibujo (Diseño), Facultad de Bellas Artes. Apdo.1397. 48080 \\ Bilbao. Bizkaia. Spain. \\ \{elena.gonzalezmiranda, tania.quindos\}@ehu.eus
}

\begin{abstract}
The basic principles of design are the essential guidelines that need to be considered at the beginning and during the stages of the Design Process. The principles related to icon systems are inferred from and defined through experience and daily practice. In this paper, we make a contribution to basic research by proposing ten principles for designing System Icons for the Graphic User Interface. These premises, applied with wise criteria and flexibility, allow to produce harmonic, consistent and effective signs for our electronic devices.
\end{abstract}

Keywords: Icons, User Interface Icons, Design Principles, Pictograms.

\section{Introduction}

In the last decades, a substantial change has taken place in the professional profile of the graphic designers out of the need of adapting our knowledge to new digital media. By changing the medium in order to organize the messages, we also deal with new projects with perceptual, conceptual and methodological challenges, such as the design of icons for the User Interface. In the 21st century, we are living a real enhancement of these small signs that are used intensively and daily in our electronic devices.

In the 1980s, designer Susan Kare made an important contribution to the study of these icons, when she designed the typographic and iconographic signs for Apple. This designer had to transform a small grid of pixels, into a family of symbols "in order to make it simple for people to use the computer" (Abdullah and Hübner, 2006).

Since then, with the same purpose of developing accessible interfaces, static icons have been designed to indicate tools or actions in computer programs; other examples are dynamic and interactive icons such as video display, scroll bar, sound icon or the different cursors to mark the position on the screen. In the last years, applications developed to be executed through smart mobile devices have also appeared. These apps use these signs as a hyperlink to access to the complexity of their contents. In these new projects, designers apply their knowledge of the design of pictograms to the design of families of screen icons. 
On the other hand, we know that basic principles are an essential knowledge in many disciplines. These guidelines allow us to begin and validate the work, with the certainty that we are on the right track.

The renowned designer Dieter Rams defined 10 principles that answer the question "Is my design good design?" For him a good design should be innovative, useful, aesthetic, understandable, honest, unobtrusive, long-lived, consistent, and environmentally friendly. "Last but not least, good design is as little design as possible (less but better)" (Dieter Rams; in Fairs, 2004).

These commandments, which recommend eliminating all unnecessary elements, can be found in the Isotype project in 1924, or in the pictograms system for the Munich Olympic Games of 1972, designed by Otl Aicher. Another significant example is the standardized pictograms of many airports in the world, developed by the American Institute of Graphic Arts (AIGA and DOT) (AIGA, 1981).

Other authors such as Abdullah and Hübner (2002), Costa (2007) or Norberto Chaves (2009), listed general principles and other recommendations to be considered when designing an iconic sign.

Within this theoretical framework, we have deemed pertinent to specify the basic rules to be taken into account at the beginning and during the Design Process of a system of icons for digital media. Icons of interactive contexts, specifically designed for this contribution, illustrate these principles.

\section{The 10 Principles for Designing Systems of Screen Icons}

\section{Articulate accurate and relevant messages}

First of all, we must carry out an inventory of the items that are really necessary and relevant to most users of the sign system. An accurate utterance will provide us with the right guidelines and will avoid errors of approach at the beginning of the design process, since the wording conditions the graphic result. (Figures 1 and 2)

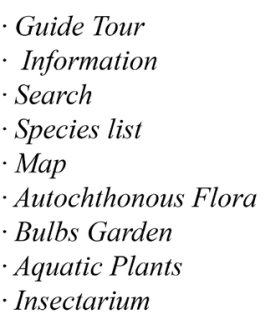

Fig. 1. Inventory for an app about a Botanical Garden.

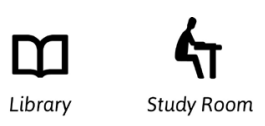

Fig. 2. At a university, the 'Study Room' does not fulfill the same function as a 'Library' and the sign on the digital signage map must reflect that difference. 


\section{Choose the appropriate iconographic referent}

Once the messages have been defined, we must select the appropriate and meaningful referent ${ }^{1}$ that will ease the interpretation and decoding of the message (Figures 3 and 4)

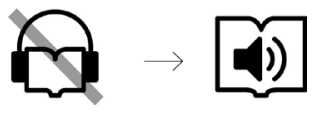

Fig. 3. In the 'audiobook' icon, it is not appropriate to represent headphones since these books can be listened without them. Speaker icon may be more meaningful.

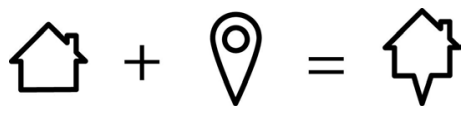

Fig. 4. In some cases, several referents can be joined resulting in composite icons. Message: I select this house on a map.

\section{Shape the sign respecting the logical physiognomic form}

The referent must have a form that corresponds to its constitution and logical structure so that the receiver identifies the object represented. (Figures 5 and 6 )
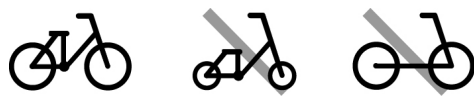

Fig. 5. Logical physiognomic form of a bicycle for a cycling route app. In the second icon, there is an obvious disproportion in some of the elements. The third sign is missing some essential features and confuses the receiver.

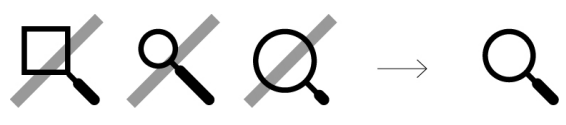

Fig. 6. Logical physiognomic shape of a magnifying glass.

\section{Adapt the referent to the context of the receiver}

By choosing current models adapted to the cultural and geographical context of the user, the understanding of the iconographic sign is facilitated. (Figures 7 and 8)

\footnotetext{
${ }^{1}$ The etymological meaning of the word "referent" is that it refers to or relates to something. In semiological terms it is the real object to which the sign alludes.
} 


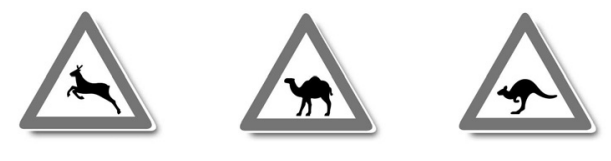

Fig. 7. As in traffic signals, on a GPS device interface, the warning message for 'Caution, wild animals' will have to be adapted to different geographic areas.

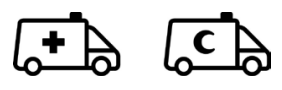

Fig. 8. On a health emergency application, 'Request an ambulance' will be represented by a cross or by the crescent, according to the country.

\section{Design by using widespread, conventional forms}

When the referent shows formal and/or stylistic variety, we will have to design a conventional and widespread form, which is found in the collective imaginary of the receiver. (Figure 9)
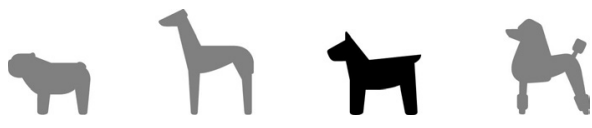

Fig. 9. On an app to know where dogs are allowed, the graphic solution should represent all dogs, regardless of race or size.

\section{Synthesize and refine the referent: Graphic sobriety}

The shape of each pictogram must be refined and reduced to its essential features. This avoids excessive and irrelevant visual information. "The sign must be saturated, that is, lacking of zones deprived of meaning. If when eliminating an element nothing is lost, is because that element was unnecessary". ${ }^{2}$ (Figure 10).

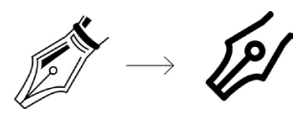

Fig. 10. The nib is reduced to its significant features.

\section{Ensure that the sign is easily learned}

The learning of a new sign must be immediate or it must occur in a very short time.

\footnotetext{
${ }^{2}$ In Chaves, Norberto (2015) Ten principles of graphic design. Foroalfa. Published on 04/15/2015. Accessed on 09/20/2017, retrieved from: https://foroalfa.org/articulos/tenprinciples-of-graphic-design
} 
Nowadays - as in the evolution of species or language - signs that conform an 'iconographic dialect' emerge or die in the continuous evolution of our contemporary culture. (Figure 11)

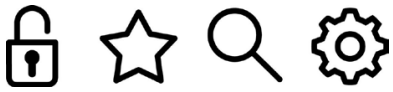

Fig. 11. Metaphors we have learned from using social networks: Privacy (padlock), Favorites (star), Search engine (magnifying glass) or Settings (cogwheel).

\section{Avoid linguistic signs}

The interpretation of alphabetic characters is conditioned by the specific language of the receiver. For this reason, we should avoid as far as possible the use of letters in a sign.

However, in some signs, the obvious referent is a number or letter and then its use is unavoidable. (Figure 12)

\section{Á̀ (i) ?}

Fig. 12. Alphabet referents to choose the size of the letter, to obtain information or to ask for help.

\section{Systematize visual constants: Formal consistency}

A system of pictograms will be perceived as a harmonic visual unit if all the elements that comprise it have a common structure or grid that guarantees its formal consistency. (Figures 13 and 14). In these visual constants, it is also necessary to establish appropriate chromatic or formal criteria to indicate the selected contents. (Figure 15)
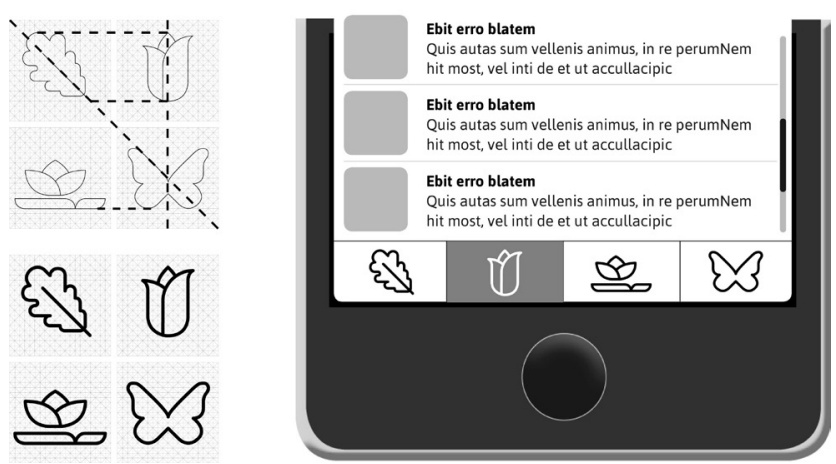

Fig. 13. The items Autochthonous Flora, Garden of Bulbs, Aquatic Plants and Insectarium of a Botanical Garden App, have been adjusted to the grid. Similar parameters have been intentionally sought in height, width or angle. 


\section{ÁABCDEFGHIJKLMN NOPORSTUWWXYZ abcolefghijklmn ñopqrstuvwxyz

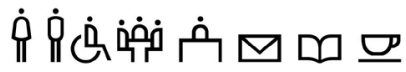 1234567890

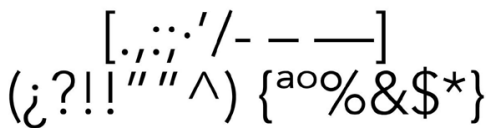

Fig. 14. Consistency and syntactic compatibility with the typographical signs of visual identity are sometimes required. In pictograms of typographic origin for the Avenir font, a recognizable visual vocabulary is established with the following characteristics: elimination of superfluous features, number of details reduced to a minimum, assignment of font proportions, lines of similar thickness in the characters, tendency to symmetry and finally, formal similarity of certain structures-like the dot with the head of the person.

\section{仓Q $\mathrm{A} Q \mathrm{Q}$}

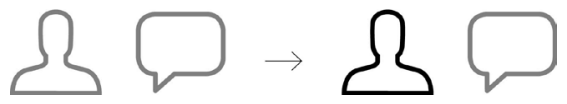

Fig. 15. The section selected on the application is represented by a filled-in shape or by a different line color.

\section{Optimize the signs for the screen}

As when designing web typographies, pictograms too have to be created so that they are optimally perceived on electronic devices' screens. On the one hand, it is necessary to make some optical adjustments so that the human eye perceives each sign in harmony within the system as a whole. It may be necessary to adjust the shape when pictograms are to be displayed in smaller sized devices. We must also adjust the vertical and horizontal strokes or check the visual weight of the connections between strokes, among other optical corrections. ${ }^{3}$ (Figures 16 y 17)

\footnotetext{
${ }^{3}$ Further information on optical adjustments on González-Miranda, E. and Quindós T. (2015) Diseño de iconos y pictogramas. Pp. 139-145.
} 
On the other hand, a smoothing process must be carried out by applying halftones on the contours of the forms. This process aims to make staggered edges uniform on monitors. (Figure 18)
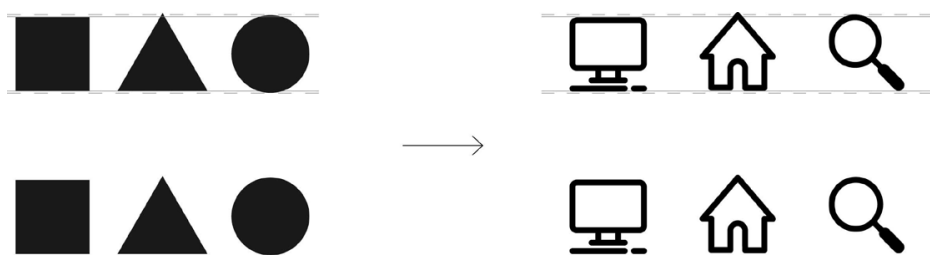

Fig. 16. Optical adjustments in a family of signs.

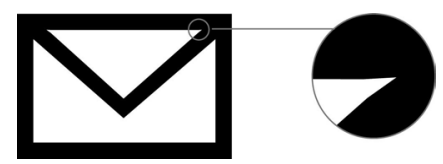

Fig. 17. Connections between strokes.

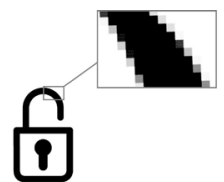

Anti-aliasing ON

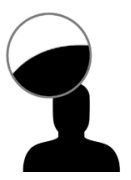

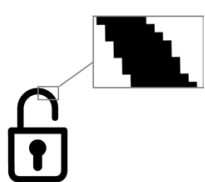

Anti-aliasing OFF

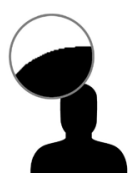

Fig. 18. Anti-aliasing process.

\section{Concluding remark}

We can summarize these ten tips in the following diagram. (Figure 19).

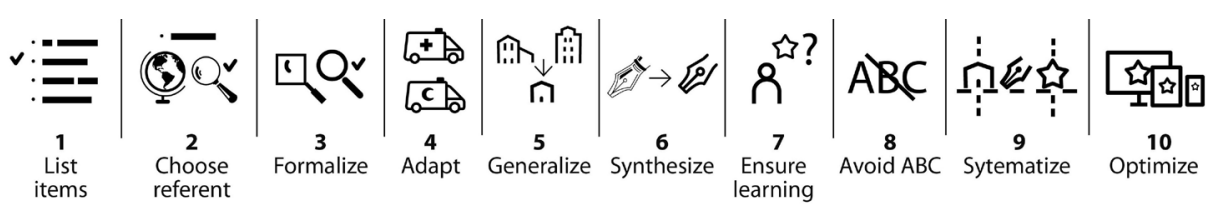

Fig. 19. Figure representing the points previously explained. 
We have to clarify that the designer has to take these principles into account throughout the design process but should not be dogmatic in the practical application of them. Designers have to be flexible enough to adapt these recommendations to the specificity of the project in hand, in order to ensure the communicative effectiveness of each sign and the consistency of the system.

\section{Acknowledgements}

This contribution has been financed in part by University of the Basque Country, UPV/EHU. Tania Quindós was granted by UPV / EHU for her Doctoral Thesis project on Pictograms and Typography.

\section{References}

Abdullah, R. and Hübner. R. (2002) Pictograms, Icons \& Signs. A guide to information Graphics. London: Ed. Thames \& Hudson Ltd.

Aicher, O. and Krampen, M. (1979) Sistemas de signos en la comunicación visual. Barcelona: Gustavo Gili SA.

American Institute of Graphic Arts, AIGA (1984) Símbolos de señalización. (Symbol Signs). Barcelona: Editorial Gustavo Gili SA.

Costa, J. (2007) Señalética corporativa. Barcelona: Costa Punto Com.

Chaves, N. (2009) Ten principles of Graphics design. 3/08/2009. Retrieved from: https://foroalfa.org/articulos/ten-principles-of-graphic-design

González-Miranda, E. and Quindós T. (2015) Diseño de iconos y pictogramas. Valencia: Campgràfic.

Fairs, M. (2004) Dieter Rams interview. Icon Magazine 10: February 2004. Retrieved from: http://web.archive.org/web/20070310142422/http://www.iconmagazine.co.uk/issues/010/rams_text.htm.

Morris, C.W. (1985) Fundamentos de la teoría de los signos (Foundations of the Theory of Signs). Barcelona: Paidós Comunicación.

Pierce, T. (1996) The International Pictograms Standard. USA: Design Pacifica Int. LLC.

Smitshuijzen, E. (2007) Signage Design Manual. Baden: Lars Müller Publishers. 